\title{
Intervenções Preventivas com Casais: $O$ que Podemos Aprender com a Experiência Internacional?
}

\author{
Aline Sardinha",1 (D) \& Terezinha Féres-Carneiro² (D) \\ ${ }^{1}$ Universidade Federal do Rio de Janeiro, Rio de Janeiro, RJ, Brasil \\ ${ }^{2}$ Pontificia Universidade Católica do Rio de Janeiro, RJ, Brasil
}

\begin{abstract}
RESUMO - A dissolução da unidade conjugal é um desafio familiar e para os diversos sistemas sociais a esta relacionados. O objetivo deste artigo é revisar a literatura sobre programas preventivos com casais, visando a identificar os formatos de sucesso, as lacunas existentes no que é oferecido no Brasil e o que podemos aprender com a experiência internacional. Os dados são apresentados e discutidos privilegiando os estudos que se mostraram teórica e empiricamente relevantes para o desenvolvimento de um programa adaptado à realidade brasileira. Os estudos encontrados apontam para a eficácia das intervenções preventivas na promoção de habilidades relacionadas à resiliência conjugal. Entretanto, os mecanismos de mudança implicados nesse processo e sua repercussão na qualidade conjugal ainda não foram esclarecidos.
\end{abstract}

PALAVRAS-CHAVE: aconselhamento conjugal, satisfação, resiliência, psicologia preventiva, terapia familiar

\section{Preventive Interventions with Couples: What Can we Learn from the International Experience?}

\begin{abstract}
The dissolution of a marital unit challenges not only the family but also a great range of social systems related to it. The article aimed to review the literature on preventive programs with couples, willing to identify the elements of success, the gaps in the interventions offered in Brazil, and summarize what we should learn from the international experience. Data will be presented and discussed, highlighting the studies found to be theoretically and empirically relevant to the development of a program targeting the prevention of crisis and the promotion of marital resilience adapted to the Brazilian reality. Results point to the efficacy of preventive interventions in promoting relational skills associated with marital resilience. However, the mechanisms of change implicated in this process, as well as its impact on marital quality, are still to be further clarified.
\end{abstract}

KEYWORDS: marriage counseling, satisfaction, resilience, preventive psychology, family therapy

Os índices de divórcio em todo o mundo vêm crescendo nos últimos anos. De acordo com dados do Instituto Brasileiro de Geografia e Estatística (IBGE, 2011), os relacionamentos conjugais no Brasil duram, em média, 15 anos, com a maior parte dos divórcios ocorrendo nos primeiros nove anos. Problemas de relacionamento entre cônjuges e de insatisfação no casamento têm sido apontados como um dos maiores estressores da vida, contribuindo para transtornos psiquiátricos e enfermidades físicas, maior mortalidade e déficits no funcionamento ocupacional e social (Sbarra, 2015). A dissolução da unidade conjugal é, portanto, um desafio não apenas para o casal, mas para diversos sistemas que estão direta ou indiretamente ligados à família nuclear: escola, empresas, igrejas, comunidade, família estendida, sistema judiciário e órgãos de assistência social.

$\mathrm{Na}$ medida em que o casamento moderno se baseia prioritariamente no amor romântico, a satisfação conjugal passa a ocupar um lugar de destaque no imaginário dos casais e os aspectos da interação adquirem um peso significativo na estabilidade e harmonia familiares (Giddens, 1993). Dessa forma, vivemos hoje um aumento da complexidade acerca da definição do que é uma vida a dois satisfatória (Wagner, Mosmann, \& Falke, 2015).

* E-mail: alinesardinhapsi@gmail.com

- Submetido: 24/05/2017; Revisado: 24/07/2019; Aceito: 13/08/2019. 
Apesar de muitas vezes tratados como sinônimos na literatura, no contexto do presente estudo, será necessário distinguir os conceitos de qualidade, ajustamento, estabilidade e satisfação conjugal. De acordo com Mosmann, Wagner e Féres-Carneiro (2006), a qualidade conjugal é a expressão de como as pessoas avaliam seus relacionamentos a cada momento. Em geral, esta é resultado da articulação de três grupos de fatores: os recursos pessoais, o contexto em que o casal está inserido e os processos adaptativos no enfrentamento de crises. $\mathrm{O}$ ajustamento conjugal, conforme definido por Spanier (1976), é uma característica dinâmica dos relacionamentos e diz respeito ao nível de concordância dos cônjuges sobre diversos temas do cotidiano familiar, à percepção sobre as discordâncias e a possibilidade de divórcio, ao compromisso e à satisfação com a relação conjugal, ao compartilhamento de interesses e perspectivas, à ausência ou presença de afetos e à relação sexual. Estabilidade conjugal, por sua vez, será aqui considerada a manutenção da conjugalidade ao longo do tempo.

A satisfação conjugal, por sua vez, é uma avaliação subjetiva do casamento, obtida após a comparação do que é percebido no casamento com os modelos e expectativas construídos sobre os casamentos a partir da sociedade e da cultura (Scorsolini-Comin \& Santos, 2009). A satisfação com o casamento se relaciona ainda com sentimentos de bemestar, contentamento, companheirismo, afeição e segurança. Tais fatores promovem intimidade no relacionamento e decorrem da congruência entre as expectativas e aspirações dos cônjuges e a realidade vivenciada (Norgren, Souza, Kaslow, Hammerschmidt, \& Sharlin, 2004). Dela Coleta (1989) ressalta também a complexidade deste fenômeno, na medida em que sofre influência de diversas variáveis como: características de personalidade, valores, atitudes, necessidades, sexo, momento do ciclo da vida familiar, presença de filhos, nível de escolaridade, nível socioeconômico, nível cultural, trabalho remunerado e experiência sexual anterior ao casamento. Conforme postulou Beck (1995), para que um casamento sobreviva e seja vivido com satisfação, são necessárias competências para além da mera existência do amor. Habilidades de interação, como empatia e expressão de sentimentos, parecem também estar intimamente relacionadas à satisfação experimentada pelo parceiro na relação (Sardinha, Falcone, \& Ferreira, 2009).

Intervenções psicoterápicas com casais em crise têm sido propostas ao longo dos anos visando a retomar a satisfação conjugal, diminuindo o impacto das crises do casal na estabilidade conjugal, nos indivíduos, no sistema familiar e nos demais sistemas a este relacionados. Entretanto, neste início de século, a literatura em psicologia e saúde mental aponta para a necessidade de os profissionais atuarem não apenas no tratamento dos transtornos psicológicos ou dos problemas familiares, através da psicoterapia, mas também na prevenção de crises conjugais, por meio de programas de intervenção especificamente voltados para esse fim (Bradley \& Hojjat, 2016; Vedes, Lind, \& Lourenço, 2011).

Considerando o modelo de medicina preventiva proposto por Leavell e Clark (1965), a prevenção pode ocorrer em três níveis: primária, sob forma de promoção de saúde ou pela implementação de medidas gerais e educativas que visam a melhorar a resistência e o bem-estar geral dos indivíduos, prevenindo eventos específicos; secundária, a partir da detecção precoce do problema a ser prevenido, gerando ações imediatas e contundentes no sentido de limitar seus efeitos nocivos; e terciária, que consiste no cuidado com os sujeitos e na recuperação das sequelas deixadas pelo problema, visando à recuperação do equilíbrio e da funcionalidade. Nesse enquadre, as ações de prevenção conjugal abordadas neste artigo, seriam ações de prevenção primária e secundária, ao passo que a psicoterapia de casal poderia ser considerada uma intervenção em nível terciário. Utilizaremos, frequentemente, os termos educação relacional e prevenção conjugal como sinônimos de promoção da satisfação e da resiliência conjugal, por serem termos largamente utilizados na literatura.

A resiliência conjugal permite que os casais enfrentem situações de estresse, minimizando seu impacto sobre a saúde física e psicológica de seus membros, bem como sobre o funcionamento individual e familiar. Essas habilidades parecem permitir que o indivíduo ou o casal tenha atitudes e comportamentos que atenuem o impacto dos eventos estressantes e potencializem o aparecimento de recursos pessoais e conjugais de enfrentamento, bem como utilizem adequadamente das fontes de apoio disponíveis (Bradley \& Hojjat, 2016).

O conceito de resiliência familiar, conforme Walsh (2005), está fundamentado na Teoria dos Sistemas, integrando perspectivas ecológicas e desenvolvimentais. O objetivo principal dessa abordagem é identificar os processos fundamentais que permitem aos casais e famílias enfrentarem crises agudas ou estressores persistentes para fortalecer seus vínculos, recuperar seu funcionamento e ir adiante em suas vidas. Walsh propõe a interação de três domínios de processos de funcionamento familiar fundamentais, que reforçam as competências de um casal para lidar efetivamente com o estresse: os sistemas de crença, os padrões organizacionais e os processos de comunicação. Uma abordagem orientada à resiliência familiar ou conjugal, de acordo com essa autora, visa a identificar e fortalecer os processos interacionais fundamentais que permitiriam à família ou ao casal resistir aos desafios desorganizadores da vida e se reestruturarem a partir deles. Essas estratégias clínicas têm como pressuposto que as habilidades e padrões que promovem resiliência podem ser desenvolvidos através de intervenções especificamente voltadas para esse fim e que teriam o potencial de prevenir e minimizar o impacto das crises.

Tais programas, conhecidos internacionalmente como educação relacional, têm como alicerces a psicoeducação 
e o desenvolvimento de habilidades de interação, comunicação, negociação, solução de problemas e regulação das emoções (Markman \& Rhoades, 2012). O objetivo deste artigo é revisar a literatura disponível no
Brasil e no exterior acerca do formato e da eficácia desses programas preventivos com casais, visando a informar futuras propostas de intervenção adaptadas às necessidades e idiossincrasias da nossa população.

\section{MÉTODOS}

A busca de literatura foi executada em duas etapas. Inicialmente foi realizada uma pesquisa prévia nas principais bases de dados científicas nas áreas de saúde e psicologia (Pubmed, BVS-Psi e PsychInfo), de modo a conhecer os autores, as teorias e os programas publicados acerca do tema, bem como definir os descritores e os balizadores da busca sistematizada da literatura. A partir dos resultados da triagem inicial, foram definidas as palavras-chave a serem utilizadas na busca dos artigos: "relationship education", "premarital education", "couple education", "marriage education", "premarital prevention", "preventive couple intervention", "healthy marriage", "couple therapy", "couple skills", "psychoeducation", "conjugal resilience, "relationship enrichment", "couple resilience", "family resilience", prevenção, psicoeducação, resiliência e "educação conjugal". Esses descritores foram combinados a descritores relativos aos construtos e desfechos comumente associados na literatura: "marital quality", "marital stability", divorce, "relationship satisfaction", “marital distress", "relationship dissolution", "relationship adjustment", "relationship functioning", "dyadic coping", "satisfação conjugal", divórcio, "ajustamento conjugal", "qualidade conjugal".

Em seguida, foram listados todos os artigos científicos publicados nessas bases, disponíveis nos idiomas português e inglês. A partir das referências citadas nesses artigos, foram triadas manualmente outras fontes sobre o assunto a serem incluídas. Realizou-se uma análise criteriosa desses artigos, sendo selecionados para inclusão neste trabalho aqueles que traziam contribuições mais relevantes acerca de questões teóricas pertinentes ao tema, do formato dos protocolos de intervenção, bem como dados empíricos sobre sua eficácia.

Como resultados da presente revisão, foram considerados todos os estudos encontrados nessas duas estratégias de busca, apresentando-se os dados sob forma de uma revisão narrativa da literatura. Nesse sentido, o foco desta revisão não é apenas sistematizar a literatura atual, uma vez que esforços interessantes já foram feitos recentemente nesse sentido (Blanchard, Hawkins, Baldwin, \& Fawcett, 2009; Hawkins, Blanchard, Baldwin, \& Fawcett, 2008; Hawkins \& Erickson, 2015; Wadsworth \& Markman, 2013). Os dados empíricos fornecidos por esses artigos nos informam quantitativamente acerca dos níveis de eficácia desses programas e serão apresentados e discutidos criticamente a partir de sua relevância teórica e da adequação metodológica dos estudos que os originaram, privilegiando aqueles que se mostrem relevantes para o desenvolvimento de um programa de promoção da resiliência conjugal adaptado à realidade brasileira.

A partir da combinação entre os resultados encontrados nas duas modalidades de busca, foram elencados os principais elementos que compõem os programas de resiliência e prevenção conjugal com maiores taxas de eficácia. A síntese dessas informações aqui apresentada teve como diretriz os desafios e potencialidades de adaptação transcultural dessas iniciativas para a realidade brasileira, de modo a poder informar a construção de iniciativas nacionais baseada em evidências científicas e na experiência internacional já descrita na literatura.

\section{RESULTADOS}

\section{Estado Atual da Prevenção e Promoção de Resiliência em Casais}

Estratégias de prevenção conjugal têm sido desenvolvidas em diversos lugares do mundo enquanto uma política pública: Estados Unidos (Stanley et al., 2014), Dinamarca (Axelsen, Brixval, Due, \& Koushede, 2014), Austrália (Halford \& Simons, 2005), China (Wang, 1991; Yao, 1990), Noruega (Thuen \& Laerum, 2005), Alemanha (Hahlweg \& Richter, 2010; Kirby \& Baucom, 2007), entre outros. O pressuposto é que os custos sociais e psicossociais do divórcio e da instabilidade familiar sobre os membros do casal, os filhos e toda a comunidade podem ser minimizados com intervenções preventivas financiadas e patrocinadas pelos governos (Dion, 2005; Halford, Markman, \& Stanley, 2008; Koppelman, 2002). Para termos uma ideia do seu alcance, nos Estados Unidos, por exemplo, entre os indivíduos casados a partir de $1990,44 \%$ reportaram ter tido acesso a alguma forma de educação conjugal antes do casamento, mais comumente oferecida por instituições religiosas, e que a simples participação estava associada a menor incidência de conflitos e divórcio, bem como a maiores níveis de satisfação conjugal e comprometimento (Markman, Rhoades, Stanley, \& Peterson, 2013; Stanley, Amato, Johnson, \& Markman, 2006).

Podemos categorizar o corpo de pesquisas empíricas existentes com programas de prevenção na literatura internacional em três tipos: (1) educação conjugal e 
sexual de jovens, com o objetivo de promover escolhas e comportamentos saudáveis e responsáveis nos relacionamentos afetivos, como forma de reduzir a ocorrência de violência entre os parceiros, a gravidez precoce e a formação de famílias instáveis; (2) intervenções buscando desenvolver habilidades associadas com resiliência em casais e famílias que estão enfrentando situações potencialmente estressantes como problemas de saúde de um dos membros da família, grandes afastamentos (como no caso das missões militares, por exemplo), estresse socioeconômico crônico (famílias em situações de risco, imigrantes etc.) ou agudo, ou etapas do ciclo de vida do casal conhecidamente desafiadoras como a chegada do primeiro filho, divórcio, recasamento, envelhecimento ou viuvez; (3) programas voltados para aprimorar a satisfação conjugal e prevenir divórcios em casais jovens ou imediatamente antes do casamento, com riscos relacionais mas ainda sem queixas importantes quanto ao seu relacionamento, numa lógica de prevenção primária, ou mesmo casais com problemas conjugais, numa intervenção que se aproxima da prevenção secundária, na fronteira com a clínica da psicoterapia de casais.

A maior parte das pesquisas sobre eficácia encontradas apresentou um desenho metodológico de avaliação pósparticipação. Esses estudos, em geral, demonstram melhoras nas habilidades ensinadas nos programas e, em alguns casos, nos indicadores de qualidade e satisfação conjugal (Markman \& Rhoades, 2012). Uma série de trabalhos também vêm sendo realizados visando a avaliar os efeitos de elementos específicos da intervenção nos resultados alcançados e tentando estabelecer uma articulação teóricoprática que permita compreender e prever quais destes são responsáveis pelos efeitos mais significativos (Halford, Markman, Kline, \& Stanley, 2003).

Atualmente, encontramos na literatura estudos retrospectivos e prospectivos, visando a estabelecer associações entre variáveis como traços de personalidade, habilidades de solução de problemas, habilidades de comunicação interpessoal e resiliência, bem como satisfação conjugal. De forma geral, as variáveis dependentes são medidas através de inventários específicos ou indiretamente, por meio de desfechos como índices de divórcio e estabilidade conjugal. Outro importante campo de pesquisas é a busca dos fatores mediadores das mudanças observadas, ou seja, em determinar como os programas de prevenção e promoção de resiliência promovem mudança na satisfação e no ajustamento conjugal, na estabilidade familiar, na parentalidade eficiente e nos índices de divórcio.

Nos últimos 15 anos, pesquisas foram publicadas relatando os resultados de ensaios clínicos controlados e randomizados com intervenções visando a promover resiliência conjugal e familiar, prevenindo crises nesses contextos (Blanchard et al., 2009; Hawkins et al., 2008). Os grupos controle geralmente são submetidos a nãointervenção (lista de espera) ou a intervenções alternativas consideradas placebo. Os dados, de forma geral, apontam para um tamanho de efeito significativo das intervenções preventivas em relação às intervenções-controle nos indicadores de qualidade conjugal mais estudados: comunicação, manejo de conflitos e satisfação conjugal (Hawkins et al., 2008).

Estudos recentes têm também tentado elucidar os mecanismos de mudança presentes nesses programas e sua relação com as variáveis estudadas, especialmente aquelas que apenas serão conhecidas em longo prazo (por exemplo, taxa de divórcio e repercussões nos filhos). Desvendar o papel desses fatores mediacionais é relevante como forma de definir os elementos fundamentais para a proposição de novas iniciativas de intervenção (Wadsworth \& Markman, 2013). Podemos encontrar ainda estudos de seguimento de alguns anos após a participação nesses programas, que apontam que muitos dos ganhos obtidos tendem a se manter ao longo do tempo (Schilling et al., 2003; Stanley et al., 2010; Wood et al., 2010), especialmente nos programas que incluíam sessões de revisão no seguimento (Braukhaus, Hahlweg, Kroeger, Groth, \& Fehm-Wolfsdorf, 2003).

\section{Aspectos Formais dos Programas Existentes na Literatura}

Em termos do formato, os programas de prevenção conjugal encontrados costumam ter duração de aproximadamente doze horas, com sessões semanais ou em formatos intensivos (um final de semana, p. ex., Kotrla \& Dyer, 2008). Os dados da literatura apontam que programas com duração entre nove e vinte horas costumam oferecer melhores resultados (Hawkins, Amato, \& Kinghorn, 2013). Podem ainda ser realizados presencialmente ou online (Duncan, Steed, \& Needham, 2009), sem que tenham sido encontradas diferenças nos resultados alcançados pelos formatos presenciais, online (Braithwaite \& Fincham, 2007; Duncan et al., 2009).

Interessantemente, os programas de prevenção encontrados na literatura foram aplicados em diferentes formatos: grupos de casais, a casais isoladamente ou mesmo a grupos de indivíduos casados sem a presença do cônjuge .Entretanto, o formato em grupo parece ser mais interessante do que o individual ou com foco em um casal, gerando maiores ganhos em habilidades de comunicação positiva (Quirk, Strokoff, Owen, France, \& Bergen, 2014). Além disso, não foram encontradas na literatura diferenças nos resultados alcançados por participantes no primeiro ou segundo casamento (Lucier-Greer, Adler-Baeder, Harcourt, \& Gregson., 2014, apesar de a grande maioria dos sujeitos que tem acesso a intervenções preventivas estar no primeiro casamento ou antes dele (Doss, Rhoades, Stanley, Markman, \& Johnson, 2009).

A maior parte costuma ser oferecida por instituições públicas como o os serviços de saúde, centros comunitários, o exército americano e organizações religiosas. Tais 
intervenções raramente são oferecidas por psicoterapeutas, tendo como líderes ou facilitadores frequentemente capelães ou pessoas especificamente treinadas naquele programa. Isso não produz resultados diferentes dos alcançados quando os programas são conduzidos em universidades, por profissionais (Markman et al., 2013).

Apesar de o corpo de evidências derivar de amostras com casais heterossexuais, brancos e de classe média, nos últimos anos, tem crescido a ampliação da oferta de programas e os estudos de eficácia em outras populações, como de casais homossexuais (Whitton, Weitbrecht, Kuryluk, \& Hutsell, 2016), de famílias recasadas (Garneau \& Adler-Baeder, 2015; Lucier-Greer et al., 2014), de população de baixa renda (Williamson, Altman, Hsueh, \& Bradbury, 2016), de imigrantes (Quirk et al., 2014), de casais parentais separados (Wood, McConnell, Moore, Clarkwest, \& Hsueh, 2010), de população encarcerada (Harcourt, Adler-Baeder, Rauer, Pettit, \& Erath, 2015). Reflete-se, assim, a diversidade dos arranjos conjugais e familiares presentes na sociedade. Entretanto, tais estudos são iniciativas isoladas e os efeitos encontrados, apesar de positivos e estatisticamente significativos, não são ainda expressivos (Hawkins \& Erickson, 2015).

Em geral, os programas de prevenção e promoção de habilidades de resiliência em casais são eficazes tanto como uma estratégia de prevenção universal, com participantes em relacionamentos que geralmente estão funcionando bem, quanto como uma estratégia de prevenção específica para casais cujos relacionamentos estão em risco ou passando por momentos de crise (Quirk et al., 2014; Rauer et al., 2014). Por outro lado, um estudo recente mostrou também que participar em programas de educação conjugal não preveniu o declínio da satisfação conjugal em recém-casados. A hipótese levantada pelos autores é a baixa qualidade dos programas oferecidos, principalmente quando consideramos que são ministrados por facilitadores leigos ou religiosos (Cobb \& Sullivan, 2015). Os tamanhos de efeito dessas intervenções costumam ser mais fortes imediatamente após a participação, no seguimento em longo prazo de casais que funcionam bem e no seguimento em curto prazo para casais em risco (Blanchard et al., 2009).Dados atuais apontam que a intervenção pré-marital com casais sem queixas aumenta a chance de estes virem a procurar psicoterapia de casal no futuro, ao perceberem um declínio em seus níveis de satisfação conjugal (Williamson, Hammet, Ross, Karney, \& Bradbury, 2018).

\section{Principais Programas de Prevenção e Promoção de Resiliência Conjugal Existentes}

As iniciativas de educação conjugal são uma prática corrente há muito tempo nas sociedades, tendo passado a ser objeto da ciência mais recentemente. A partir da década de 1970, o amadurecimento das teorias psicológicas interacionais, aliado ao conhecimento sobre os efeitos deletérios da separação conjugal, fomentou iniciativas de promover a satisfação conjugal como um veículo para a estabilidade familiar e a prevenção do divórcio (Wagner et al., 2015). Dessa época, podemos destacar estudos como o Couples Communication (Miller, Nunnally, \& Wackman, 1976) e o Relationship Enhancement (Guerney, 1977).

Apesar do crescimento abrupto do número de estudos sobre o tema na literatura, a maior parte dos dados disponíveis se refere aos resultados de um pequeno grupo de projetos de prevenção conjugal, conduzidos em larga escala nos Estados Unidos, e seus desdobramentos: o Prevention and Relationship Enhancement Program (PREP; Stanley et al., 2014), com os militares do exército americano; o Building Strong Families Study, também financiado pelo governo americano, que teve como alvo casais parentais que não são casados, conduzido em oito locais diferentes, atingindo milhares de participantes (Wood, McConnell, Moore, Clarkwest, \& Hsueh, 2010) e, mais recentemente, o Healthy Marriage Initiative (Dion, 2005; Williamson et al., 2016).

O resultado mais impactante do PREP foi a redução do risco de divórcio à metade nos casais de militares participantes (Stanley, Allen, Markman, Rhoades, \& Prentice, 2010). Esse modelo de intervenção é o que gerou mais desdobramentos, com diversos estudos sendo reportados utilizando a versão original ou modelos adaptados a partir desta: Our Relationship Program (Doss et al., 2016), E-PREP (Hawkins \& Erickson, 2015), Strong Bonds (Allen, Stanley, Rhoades, \& Markman, 2015). A despeito do grande investimento, o Building Strong Families não apresentou resultados significativos quanto ao seu objetivo principal de favorecer estilos parentais saudáveis (Wood et al., 2010). Interessantemente, um estudo recente com o PREP for Strong Bonds apontou que, apesar da redução na taxa de divórcios alcançada, os índices de satisfação conjugal não se alteraram significativamente (Allen et al., 2015). Além disso, não há evidências de que os ganhos obtidos nesse aspecto se mantenham no seguimento de dois anos, apesar de os participantes apresentarem uma taxa significativamente menor de divórcios nesse período (Stanley et al., 2014).

O Couples Coping Enhancement Training é um programa com foco em estratégias de enfrentamento e suporte comuns para o casal, que atingiu resultados positivos na satisfação conjugal tanto na pós-intervenção quanto nos dois anos subsequentes (Pihet et al., 2007). O Within My Reach tem como alvo pessoas que não estão em relacionamentos, visando a fomentar escolhas e comportamentos saudáveis na estruturação dos casais e diminuir a violência conjugal e familiar (Rhoades \& Stanley, 2011). Derivado deste, o Within Our Reach tem como foco casais e apresentou resultados positivos tanto no pós-intervenção quanto no seguimento de 30 meses, em um estudo recente (Rhoades, 2015).O Relationship Evaluation (RELATE) e o Couple CARE (Halford et al., 2003), são programas em formato autoguiado, com o foco na comunicação entre os membros 
do casal. O Power of Two é uma versão inteiramente online de educação conjugal (Kalinka, Fincham, \& Hirsch, 2012). O Family Foundations (Feinberg \& Kan, 2008), o Working Together Program (Owen \& Rhoades, 2012 ) e uma versão adaptada do Couple CARE para pais (Petch, Halford, Creedy, \& Gamble, 2012a) têm também sido aplicados a casais parentais buscando desenvolver estilos parentais mais saudáveis e, consequentemente, alcançar melhores desfechos para os filhos.

No Brasil, é comum encontrar grupos de casais vinculados a instituições religiosas. $\mathrm{O}$ único programa de educação conjugal estruturado encontrado em nossa revisão foi uma iniciativa desenvolvida na PUCRS denominada Viver a dois. Esse programa psicoeducativo consiste em seis oficinas que buscam ampliar o leque de estratégias utilizadas no enfrentamento de conflitos e discutir temas importantes para a vida dos casais, como expectativas e mitos da conjugalidade, sexualidade e lazer (Wagner, Mosmann, \& Falcke, 2015). Resultados iniciais desse protocolo já se encontram disponíveis e apontam para uma redução dos indicadores de conflito conjugal nos casais pesquisados, apesar da ausência de impacto significativo na qualidade conjugal (Neumann, Wagner, \& Remor, 2018). Além deste, poucas iniciativas preventivas isoladas foram localizadas como, por exemplo, a intervenção psicoeducativa com um casal na transição para a parentalidade (Murta, Rodrigues, Rosa, \& Paulo, 2012). Dessa forma, nossa revisão de literatura apontou que, apesar de esse ser ainda um campo incipiente em nosso país, muito já foi feito em termos de programas de educação conjugal fora do Brasil.

\section{Elementos-Chave das Intervenções Existentes}

A maior parte dessas intervenções consiste em programas psicoeducativos, baseados no desenvolvimento de habilidades de comunicação, autorregulação das emoções, solução de problemas e no desenvolvimento de conexões positivas e de um senso de coesão familiar. O foco da intervenção vem, assim, aos poucos se deslocando apenas da estabilidade para a promoção da satisfação conjugal (Wagner et al., 2015). Entretanto, o ponto fundamental da grande maioria dos programas costuma ser ainda basicamente o desenvolvimento e aprimoramento de habilidades de comunicação.

Os dados da literatura mostram que, após a participação, os indivíduos têm um desempenho significativamente melhor em relação à comunicação (Blanchard et al.,
2009), bem como em habilidades de solução de problemas (Hawkins et al., 2008). Por outro lado, esse ganho não necessariamente se relaciona com maiores índices de satisfação conjugal (Williamson et al., 2016).Uma pesquisa mostrou ainda que não houve diferenças nem nos padrões negativos de comunicação nem no ajustamento conjugal nos resultados de participantes do PREP convencional, com foco em comunicação, em relação aos que receberam o mesmo programa, mas sem a instrução formal em habilidades comunicacionais(Owen, Manthos, \& Quirk, 2013). Isso aponta para uma direção importante, que vem sendo considerada nos programas mais modernos, de que talvez a melhora da comunicação, por si só, não tenha um impacto tão determinante quanto se pensava teoricamente na satisfação dos casais com o seu relacionamento (Hawkins \& Erickson, 2015).

A ênfase em comunicação deriva das influências teóricas cognitivo-comportamentais e sistêmicas na terapia de casais (Gottman \& Silver, 2012; Markman, Rhoades, Stanley, Ragan, \& Whitton, 2010). Ainda utilizando esses mesmos referenciais teóricos, alguns programas buscam também alinhar as expectativas dos membros do casal com informação psicoeducativa sobre como os relacionamentos funcionam e dados de pesquisas sobre a realidade dos casais (p. ex., Schulz, Cowan, \& Cowan, 2006). Outros enfocam ainda habilidades como perdão interpessoal e empatia (p. ex., Ripley \& Worthington, 2002).

Os programas que enfatizam a comunicação apresentam vantagens óbvias em termos de incrementar a comunicação positiva e reduzir a comunicação negativa. Evidências apontam também para a possibilidade de ênfase na comunicação positiva e no comportamento de construção da amizade produzir efeitos maiores nas habilidades de gerenciamento de conflitos do que uma ênfase na diminuição da comunicação negativa (Gottman, Ryan, Swanson, \& Swanson, 2005). Propostas recentes têm incluído, ademais, técnicas de manejo de conflito e regulação emocional, especialmente com o objetivo de reduzir a violência e os comportamentos agressivos no casamento (Kirby \& Baucom, 2007), bem como ações voltadas para a promoção do senso de compromisso (Rhoades, Stanley, \& Markman, 2009).

Além disso, variações tem sido propostas, com programas envolvendo intervenções baseadas em mindfulness (Gambrel \& Piercy, 2015), intervenções individuais visando a melhorar o relacionamento do casal (Visvanathan, Richmond, Winder, \& Koenck, 2015) e ainda programas que visam a fortalecer sujeitos em relacionamentos abusivos e violentos para deixar seus parceiros (Rhoades \& Stanley, 2009).

\section{DISCUSSÃO}

Estratégias focadas em prevenção e na promoção de resiliência têm como objetivo fazer com que os casais mantenham a satisfação conjugal ao longo do tempo. Assim, a pesquisa nesse campo é uma tarefa árdua, na medida em que tenta compreender o que, na trajetória dos casais participantes, faria com que eles tivessem um desfecho 
diferente dos casais que não passaram pela intervenção. Os resultados dos estudos aqui revisados apontam para a eficácia das intervenções preventivas na promoção da resiliência conjugal em curto prazo. Entretanto, os mecanismos de mudança implicados e o processo que faz com que a aquisição de habilidades se transforme em qualidade conjugal ainda necessitam ser mais bem esclarecidos (Wadsworth \& Markman, 2013).

Por se tratar de iniciativas recentes, os dados acerca da permanência desses efeitos no longo prazo são também escassos. Em geral, as pesquisas apontam para uma atenuação dos efeitos ao longo do tempo (Bodenmann, Pihet, \& Kayser 2006) e alguns autores propõem sessões de revisão periódicas, visando a estabilizar os ganhos obtidos no seguimento (Braukhaus et al., 2003). Considerando que os efeitos de intervenções preventivas são necessariamente de longo prazo, especialmente aquelas voltadas para ampliar a duração dos relacionamentos e diminuir a incidência de divórcio, esta é ainda uma lacuna importante do conhecimento (Hawkins et al., 2008). Além disso, é possível que os efeitos na satisfação conjugal sejam perceptíveis apenas para casais com baixa satisfação conjugal inicial e não em casais sem queixas (Halford et al., 2017).

Na psicoterapia, os problemas e mecanismos terapêuticos utilizados para tratá-los se colocam de maneira mais clara (Wadsworth \& Markman, 2013). Na prevenção, por outro lado, os efeitos podem se manifestar não ao final do programa, mas décadas depois, já que as habilidades adquiridas permitem que os membros do casal desenvolvam proficiência no uso das técnicas, aprendendo a inocular o estresse e se tornando mais resilientes ao longo do tempo, após superarem diversos momentos de crise (Neff \& Broady, 2011). Além disso, a literatura aponta que a simples participação é, em si, um elemento de resiliência conjugal, ao aumentar a probabilidade com que esses casais tendem a buscar ajuda profissional ou fazer psicoterapia de casal no longo prazo. É possível que participar de uma ação preventiva fortaleça a crença de que existem habilidades que podem ser aprendidas e medidas que podem ser tomadas para melhorar o relacionamento. Dessa forma, esses casais sabem que existe ajuda disponível e onde procurá-la quando isso se tornar necessário no futuro (Williamson, Hammett, Ross, Karney, \& Bradbury, 2018).

Halford et al. (2003) destacam uma homogeneidade no campo da educação conjugal, no sentido que os programas costumam ter estrutura, objetivos e fundamentos teóricos semelhantes. O foco principal consiste na aquisição de habilidades de comunicação em um estilo didático, comumente em grupos e em ambientes como universidades, igrejas e centros comunitários. Além disso, os resultados medidos costumam ser também os mesmos: habilidades comunicacionais, satisfação conjugal e estabilidade familiar. Essa homogeneidade não representa, contudo, uma hegemonia de práticas escolhidas a partir de evidências científicas, mas um pensamento unificado do pequeno grupo que atua na área (Hawkins et al., 2008). Seria importante, então, ampliar a oferta de programas que incluíssem outras questões já levantadas pela literatura de psicoterapia de casal, como senso de propósito e valores dos membros do casal e suas crenças sobre a importância do casamento e da família. Tais aspectos podem ser tão importantes para a qualidade e a estabilidade de relacionamento quanto as habilidades de comunicação (Fincham, Stanley, \& Beach, 2007).

Por outro lado, quando a comunicação melhora, é comum que outros processos como proximidade, coesão e senso de propósito melhorem também (Heatherington, Friedlander, \& Greenberg, 2005), deixando a dúvida de que elementos seriam de fato os principais agentes responsáveis pela eficácia dos programas em termos da qualidade conjugal (Wadsworth \& Markman, 2013). Além disso, a maior parte dos estudos aponta para uma diminuição da comunicação disfuncional ao invés de um incremento da comunicação positiva. Nesse sentido, Markman et al. (2010) sugerem ampliar o foco de comunicações positivas para trocas positivas de uma forma geral, intensificando momentos de diversão conjuntos, a amizade, o romance, a intimidade e a sexualidade. O campo é, portanto, fértil para iniciativas criativas que articulem diferentes propostas teóricas de modo a alcançar resultados mais promissores, bem como compreender melhor os mecanismos de mudança implicados em sua eficácia (Hawkins et al., 2013).

Uma lacuna importante na literatura está relacionada a ações preventivas no campo da sexualidade dos casais. Apesar do papel fundamental que o sexo ocupa na estruturação do casal, e no funcionamento psicológico humano, na vasta produção existente em psicoterapia de casal nas diferentes abordagens teóricas, poucos são os textos especificamente voltados para esse tema. Ainda que frequentemente mencionado, o aspecto sexual do relacionamento conjugal, especificamente, acaba, muitas vezes, sendo negligenciado tanto na prática clínica quanto na produção científica na área (Carvalho \& Sardinha, 2017). Esta revisão mostrou que o mesmo cenário se repete no campo da prevenção conjugal. Alguns estudos, que têm como alvo jovens, trabalham a sexualidade no sentido de práticas responsáveis de planejamento familiar e contracepção. Entretanto, nenhum programa encontrado desenvolve educação sexual no sentido de aprimorar a satisfação sexual do casal.

Esse é um ponto negligenciado que precisa ser incluído, dado que insatisfação com a vida sexual é um grande preditor de insatisfação conjugal. Uma pesquisa envolvendo 387 casais casados descobriu que tanto a satisfação sexual quanto a comunicação entre os membros do casal estavam intimamente relacionadas à satisfação com o casamento (Litzinger \& Gordon, 2005). Porém, cada uma dessas variáveis contribuía para a satisfação conjugal de forma independente. De acordo com os autores, casais que tinham uma comunicação adequada, mesmo sexualmente 
insatisfeitos, apresentavam níveis adequados de satisfação com o relacionamento. Por outro lado, dificuldades de comunicação parecem ter menor impacto na insatisfação com o casamento em casais sexualmente satisfeitos. Um estudo recente mostrou, inclusive, que mesmo altos níveis de intimidade emocional entre os cônjuges não parecem ter um efeito direto sobre a satisfação sexual reportada por eles (Yoo, Bartle-Haring, Day, \& Gangamma, 2014).

Há também evidências incipientes de que a participação nesses programas pode impactar positivamente o desfecho de casais com níveis moderados de estresse conjugal ou em momentos sensíveis do ciclo de vida (Halford et al., 2015). Um estudo qualitativo sobre o programa com pessoas que participaram do PREP e acabaram por se divorciar aponta que o programa poderia melhorar nos seguintes aspectos: participação antes de assumir o compromisso de se casar, quando seria mais fácil desistir daquele relacionamento, suporte para implementar as técnicas fora do programa e um maior foco sobre as mudanças no casamento ao longo do ciclo vital do casal (Scott, Rhoades, Stanley, Allen, \& Markman, 2013).

Nesse sentido, é importante notar que os resultados desejáveis para os diversos perfis de casais participantes podem ser distintos. Para os casais já satisfeitos, a ideia é prevenir a deterioração da qualidade conjugal ao longo do tempo, enquanto, para os casais em situações de maior risco, o foco estaria em promover habilidades de resiliência e conseguir impactar positivamente a maneira como manejam a adversidade. Para indivíduos em relacionamentos abusivos ou violentos, por exemplo, o divórcio poderia ser considerado, inclusive, um desfecho positivo. Então, devemos avaliar os resultados esperados à luz das necessidades do grupo a ser incluído na intervenção,

Há ainda controvérsia acerca de se as intervenções preventivas devem se manter psicoeducativas ou incorporar aspectos clínicos, especialmente em função da participação cada vez maior de casais com diferentes perfis e demandas. Ao entendermos a psicoterapia como uma ação voltada para momentos de crise, visando a atenuar o estresse e melhorar a qualidade conjugal, é possível que ferramentas clínicas já empiricamente validadas possam ser incluídas como forma de potencializar os resultados nessa população, atuando como estratégia de prevenção secundária ou terciária. Além disso, enquanto os programas preventivos têm como objetivo aprimorar habilidades que poderão ser aplicadas a problemas em geral, a psicoterapia costuma tratar do conteúdo dos temas de conflito, o que pode ser relevante para situações em que as crenças e os valores dos membros do casal são muito discrepantes, impactando significativamente o ajustamento conjugal (Halford et al., 2003).Entretanto, o estigma social da psicoterapia de casais como algo de que só participam os casais com graves conflitos é um desafio à inclusão maciça de indivíduos fora de situações de crise. Nesse sentido, a proposta de educação conjugal e prevenção parece ser mais palatável do que a de psicoterapia, em função do foco no aprendizado de habilidades ao invés da discussão dos temas de conflito (Ooms, 2010).

Ao contrário da psicoterapia, contudo, os programas preventivos costumam ser estruturados, o que consiste em uma vantagem ao permitir que profissionais que não são psicoterapeutas possam ser treinados especificamente como facilitadores. Essa é uma possibilidade interessante, dado que não dispomos em nosso país de profissionais suficientes para atender à atual demanda de psicoterapia de casais, menos ainda para realizar intervenções preventivas regularmente com casais fora da clínica. Porém, a proposta de incorporar elementos da psicoterapia nos programas demandaria ainda mais profissionais capacitados, além de esbarrar nos limites legais da prática da psicoterapia no Brasil. Dessa forma, se por um lado isso poderia intensificar os efeitos obtidos e solucionar algumas das lacunas levantadas pela literatura de modo a potencializar o impacto destas intervenções; por outro, talvez perdêssemos em alcance, dada a dificuldade de contar com facilitadores aptos nesse sentido.

Uma dificuldade constantemente apontada na literatura é motivar pessoas que não estão passando por situações de crise a participarem dos programas preventivos, notadamente os homens (Doss et al., 2009). É possível ainda que a sensibilização dos casais para a participação ou mesmo o emprego de técnicas breves de promoção de resiliência conjugal possam ser facilitadas em momentos de vida em que estes utilizam os serviços públicos com mais intensidade, como na chegada de um filho, no adoecimento ou em situações de vulnerabilidade socioeconômica. Nesse sentido, intervenções preventivas poderiam ser incorporadas em programas já estruturados no Brasil como o Programa de Saúde da Família, nos serviços de saúde, nas escolas e nos centros comunitários, através do treinamento de profissionais de saúde e assistência social para atuarem como facilitadores.

O treinamento dos psicólogos de forma geral pode permitir ainda que os casais possam ser avaliados e treinados em habilidades de forma breve, quando têm contato com algum profissional de psicologia nos diferentes campos de atuação do psicólogo: hospital, empresa, escola e clínica particular. Esses momentos poderiam funcionar como janelas de oportunidade para a inclusão de programas que desenvolvam habilidades preventivas e de resiliência nos serviços assistenciais já utilizados ou para o encaminhamento para a participação em um programa em outra instituição (Petch, Halford, Creedy, \& Gamble, 2012b). 


\section{CONSIDERAÇÕES FINAIS}

A forma como os casais lidam com os desafios da vida pode influenciar consideravelmente o bem-estar imediato e de longo prazo da unidade familiar e de cada um dos seus membros. As pesquisas voltadas para a resiliência e para prevenção buscam compreender as maneiras pelas quais os profissionais podem identificar, ratificar e fortalecer as habilidades que transformam a maneira como os casais lidam com as adversidades, visando a manter a satisfação conjugal e a estabilidade familiar. O pressuposto é que tanto a resiliência individual quanto a familiar podem ser forjadas por esforços cooperativos e pelo desenvolvimento de habilidades para lidar com os estressores.

Entretanto, a questão a ser solucionada é como transpor os ganhos alcançados na proficiência dessas habilidades em satisfação conjugal, melhora da qualidade dos relacionamentos e, consequentemente, menos divórcios e instabilidade familiar. O pressuposto é que aprender determinadas habilidades e princípios e utilizá-los no momento de crise levaria imediatamente a um melhor funcionamento do casal, prevenindo problemas futuros no relacionamento. Apesar de razoável do ponto de vista teórico, tal hipótese não se confirma nos testes empíricos de forma confiável. Outro aspecto importante é que da forma como os estudos de eficácia foram desenhados, com avaliações pré e pós-participação, é difícil distinguir se a aquisição de habilidades é um resultado em si ou um mecanismo para alcançar a qualidade conjugal. De qualquer forma, uma lente de resiliência e prevenção muda a perspectiva sobre as famílias e os casais em situações de angústia, concebendo-os como desafiados e focalizando seu potencial de reparo e crescimento.

No Brasil e no mundo, a maior parte dos casais que poderia se beneficiar de psicoterapia não tem acesso a esse tipo de tratamento tanto por questões sociais que estigmatizam a busca de terapia de casal, quanto por dificuldades financeiras e pela indisponibilidade de profissionais qualificados. Tais intervenções breves preventivas podem ser uma alternativa para a promoção da saúde relacional das famílias e a redução do risco social e psicológico associado ao divórcio. De maneira geral, esses programas apresentam vantagens como o largo alcance e a curta duração. Os dados apontam ainda que fatores culturais medeiam a eficácia desses programas e que os mesmos devem ser adaptados ao contexto cultural e socioeconômico em que os casais se encontram.

$\mathrm{O}$ fato de as intervenções serem ministradas por profissionais especificamente treinados é um caminho promissor para sua implementação em larga escala. Por outro lado, o treinamento dos psicólogos e psicoterapeutas generalistas em ações preventivas e promoção de resiliência pode ser outra alternativa interessante, permitindo que estes estejam alertas na sua prática para as questões que impactam a estabilidade familiar. Um treinamento em estratégias simples de triagem e promoção de satisfação conjugal para os psicólogos em geral poderia, por si só, aumentar a probabilidade de os casais com demandas terem acesso a algum tipo de intervenção.

A metodologia empregada neste estudo privilegiou reportar qualitativamente os esforços de prevenção conjugal existentes, salientando seus aspectos formais e objetivos, bem como refletir os resultados publicados sobre eles na literatura internacional. Nesse sentido, os dados aqui descritos são limitados no sentido de discutir o estado da arte das intervenções em prevenção conjugal e de descrever dados empíricos a estas relacionados. Assim, esta revisão pode consistir em um ponto de partida para elencar os elementos fundamentais que a literatura aponta serem relevantes de modo a construir ferramentas simples que permitam intervenções preventivas eficazes tanto voltadas para profissionais de psicologia, como para outros formatos que pudessem ser administrados por facilitadores especificamente treinados. Contudo, este é um campo ainda pouco explorado em nosso país e o que foi revisado aqui pode informar futuras iniciativas de pesquisa e intervenção preventiva com casais brasileiros, adaptando transculturalmente os programas já existentes ou construindo novas propostas que visem a enfrentar os desafios encontrados na realidade do nosso país.

\section{REFERÊNCIAS}

Allen, E., Stanley, S., Rhoades, G., \& Markman, H. (2015). PREP for Strong Bonds: A review of outcomes from a randomized clinical trial. Contemporary Family Therapy, 37(3), 232-246. https://doi.org/10.1007/s10591-014-9325-3

Axelsen, S. F., Brixval, C. S., Due, P., \& Koushede, V. (2014). Integrating couple relationship education in antenatal education - A study of perceived relevance among expectant Danish parents. Sexual \& Reproductive Healthcare: Official Journal of the Swedish Association of Midwives, 5(4), 174-175. https:// doi.org/10.1016/j.srhc.2014.06.006

Blanchard, V. L., Hawkins, A. J., Baldwin, S. A., \& Fawcett, E. B. (2009). Investigating the effects of marriage and relationship education on couples' communication skills: A meta-analytic study. Journal of Family Psychology, 23(2), 203-214. https:// doi.org/10.1037/a0015211

Bradley, J. M., \& Hojjat, M. (2016). A model of resilience and marital satisfaction. The Journal of Social Psychology, 157(5),588-601. https://doi.org/10.1080/00224545.2016.12 54592

Braithwaite, S., \& Fincham, F. (2007). ePREP: Computer based prevention of relationship dysfunction, depression and anxiety. Journal of Social and Clinical Psychology, 26, 609-622. https:// doi.org/10.1521/jscp.2007.26.5.609

Beck, A. T. (1995). Para além do amor. Rio de Janeiro: Record 
Bodenmann, G., Pihet, S., \& Kayser, K. (2006). The relationship between dyadic coping and marital quality: A 2-year longitudinal study. Journal of Family Psychology, 20(3), 485493. http://doi.org/10.1037/0893-3200.20.3.485

Braukhaus, C., Hahlweg, K., Kroeger, C., Groth, T., \& FehmWolfsdorf, G. (2003). The effects of adding booster sessions to a prevention-training program for committed couples. Behavioral and Cognitive Psychotherapy, 31(3), 325-336. https://doi.org/10.1017/S1352465803003072

Carvalho, A. C., \& Sardinha, A. (2017). Terapia Cognitiva Sexual: Uma proposta integrativa na psicoterapia da sexualidade (1st ed.). Rio de Janeiro: Cognitiva.

Cobb, R. J., \& Sullivan, K. T. (2015). Relationship education and marital satisfaction in newlywed couples: A propensity score analysis. Journal of Family Psychology, 29(5), 667-678. https://doi.org/10.1037/a0039580

Dela Coleta, M. F. (1989). A medida da satisfação conjugal: Adaptação de uma escala. Psico, 18(2), 90-112.

Dion, M. R. (2005). Healthy marriage programs: Learning what works. The Future of Children, 15(2), 139-56. Retrieved from http://www.ncbi.nlm.nih.gov/pubmed/16158734

Doss, B. D., Cicila, L. N., Georgia, E. J., Roddy, M. K., Nowlan, K. M., Benson, L. A., \& Christensen, A. (2016). A randomized controlled trial of the web-based OurRelationship program: Effects on relationship and individual functioning. Journal of Consulting and Clinical Psychology, 84(4), 285-96. https:// doi.org/10.1037/ccp0000063

Doss, B. D., Rhoades, G. K., Stanley, S. M., Markman, H. J., \& Johnson, C. A. (2009). Differential use of premarital education in first and second marriages. Journal of Family Psychology, 23(2), 268-273. https://doi.org/10.1037/a0014356

Duncan, S., Steed, A., \& Needham, C. M. (2009). A comparison evaluation study of web-based and traditional marriage and relationship education. Journal of Couple \& Relationship Therapy, 8, 162-180. https://doi.org/10.1080/15332690902813836.

Feinberg, M. E., \& Kan, M. L. (2008). Establishing family foundations: Intervention effects on coparenting, parent/infant well-being, and parent-child relations. Journal of Family Psychology, 22(2), 253-263. https://doi.org/10.1037/08933200.22.2.253

Fincham, F. D., Stanley, S. M., \& Beach, S. R. H. (2007). Transformative processes in marriage: An analysis of emerging trends. Journal of Marriage and Family, 69(2), 275-292. https://doi.org/10.1111/j.1741-3737.2007.00362.x

Gambrel, L. E., \& Piercy, F. P. (2015). Mindfulness-based relationship education for couples expecting their first child - Part 2: Phenomenological findings. Journal of Marital and Family Therapy, 41(1), 25-41. https://doi.org/10.1111/ jmft. 12065

Garneau, C. L., \& Adler-Baeder, F. (2015). Changes in stepparents' coparenting and parenting following participation in a community-based relationship education program. Family Process, 54(4), 590-599. https://doi.org/10.1111/famp.12133

Giddens, A. (1993). A transformação da intimidade: Sexualidade, amor e erotismo nas sociedades modernas. São Paulo: UNESP.

Gottman, J., Ryan, K., Swanson, C., \& Swanson, K. (2005). Proximal change experiments with couples: A methodology for empirically building a science of effective interventions for changing couples' interaction. Journal of Family Communication, 5(3), 163-190, https://doi.org/10.1207/ s15327698jfc0503 1

Gottman, J., \& Silver, N. (2012). What makes love last. New York: Simon \& Schuster.

Guerney, B. G. (1977). Relationship enhancement: Skill-training programs for therapy, problem prevention, and enrichment. San Francisco, CA: Jossey Bass.

Hahlweg, K., \& Richter, D. (2010). Prevention of marital instability and distress. Results of an 11-year longitudinal follow-up study.
Behaviour Research and Therapy, 48(5), 377-383. https://doi. org/10.1016/j.brat.2009.12.010

Halford, W. K., Markman, H. J., Kline, G. H., \& Stanley, S. M. (2003). Best practice in couple relationship education. Journal of Marital and Family Therapy, 29(3), 385-406. http://www. ncbi.nlm.nih.gov/pubmed/12870411

Halford, W. K., Markman, H. J., \& Stanley, S. (2008). Strengthening couples' relationships with education: Social policy and public health perspectives. Journal of Family Psychology, 22(4), 497-505. https://doi.org/10.1037/a0012789

Halford, W. K., Pepping, C. A., Hilpert, P., Bodenmann, G., Wilson, K. L., Busby, D., Larson, J., \& Holman, T. (2015). Immediate effect of couple relationship education on low-satisfaction couples: A randomized clinical trial plus an uncontrolled trial replication. Behavior Therapy, 46(3), 409-421. https://doi. org/10.1016/j.beth.2015.02.001

Halford, W. K., \& Simons, M. (2005). Couple relationship education in Australia. Family Process, 44(2), 147-159. http://www.ncbi. nlm.nih.gov/pubmed/16013742

Halford, K. W., Rahimullah, R. H., Wilson, K. L., Occhipinti, S., Busby, D. M., \& Larson, J. (2017). Four year effects of couple relationship education on low and high satisfaction couples: A randomized clinical trial. Journal of Consulting and Clinical Psychology, 85(5), 495-507. https://doi.org/10.1037/ ccp0000181

Harcourt, K. T., Adler-Baeder, F., Rauer, A., Pettit, G. S., \& Erath, S. (2015). Relationship education for incarcerated adults. Family Process, 56(1), 75-90. https://doi.org/10.1111/famp.12164

Hawkins, A. J., Amato, P. R. \& Kinghorn, A. (2013), Are government-supported healthy marriage initiatives affecting family demographics? A state-level analysis. Family Relations, 62, 501-513. http://doi.org/10.1111/fare.12009

Hawkins, A. J., Blanchard, V. L., Baldwin, S. A., \& Fawcett, E. B. (2008). Does marriage and relationship education work? A meta-analytic study. Journal of Consulting and Clinical Psychology, 76(5), 723-734. https://doi.org/10.1037/a0012584

Hawkins, A. J., Stanley, S. M., Cowan, P. A., Fincham, F. D., Beach, S. R., Cowan, C. P., Rhoades, G. K., Markman, H. J.,\& Daire, A. P. (2013). A more optimistic perspective on governmentsupported marriage and relationship education programs for lower income couples. American Psychologist, 68(2),110-111. https://doi.org/10.1037/a0031792.

Hawkins, A. J., \& Erickson, S. E. (2015). Is couple and relationship education effective for lower income participants? A metaanalytic study. Journal of Family Psychology, 29(1), 59-68. https://doi.org/10.1037/fam0000045

Heatherington, L., Friedlander, M., \& Greenberg, L. (2005). Change process research in couple and family therapy: Methodological challenges and opportunities. Journal of Family Psychology, 19, 18-27. https://doi.org/10.1037/0893-3200.19.1.18.

Instituto Brasileiro de Geografia e Estatística. (2011). Estatística de Registro Civil. Rio de Janeiro.

Kalinka, C. J., Fincham, F. D., \& Hirsch, A. H. (2012). A randomized clinical trial of online-biblio relationship education for expectant couples. Journal of Family Psychology, 26(1), 159-64. https://doi.org/10.1037/a0026398

Kirby, J. S., \& Baucom, D. H. (2007). Treating emotion dysregulation in a couples context: A pilot study of a couples skills group intervention. Journal of Marital and Family Therapy, 33(3), 375-91. https://doi.org/10.1111/j.1752-0606.2007.00037.x

Koppelman, J. (2002). Promoting marriage as welfare policy: Looking at a public role in private lives. NHPF Issue Brief, (770), 1-17. Retrieved from http://www.ncbi.nlm.nih.gov/ pubmed/11878356

Kotrla, K., \& Dyer, P. (2008). Using marriage education to strengthen military families: Evaluation of the active military life skills program. Social Work and Christianity, 35(3), 287311. 
Leavell, H., \& Clark, E. G. (1965). Preventive medicine for the doctor in his community. New York: MacGraw Hill.

Litzinger, S., \& Gordon, K. C. (2005). Exploring relationships among communication, sexual satisfaction, and marital satisfaction. Journal of Sex and Marital Therapy, 31(5), 409424. https://doi.org/10.1080/00926230591006719

Lucier-Greer, M., Adler-Baeder, F., Harcourt, K. T., \& Gregson, K. D. (2014). Relationship education for stepcouples reporting relationship instability - Evaluation of the Smart Steps: Embrace the Journey curriculum. Journal of Marital and Family Therapy, 40(4), 454-69. https://doi.org/10.1111/ jmft.12069

Markman, H. J., \& Rhoades, G. K. (2012). Relationship education research: Current status and future directions. Journal of Marital and Family Therapy, 38(1), 169-200. https://doi. org/10.1111/j.1752-0606.2011.00247.x

Markman, H. J., Rhoades, G. K., Stanley, S. M., \& Peterson, K. M. (2013). A randomized clinical trial of the effectiveness of premarital intervention: moderators of divorce outcomes. Journal of Family Psychology, 27(1), 165-172. https://doi. org/10.1037/a0031134

Markman, H. J., Rhoades, G. K., Stanley, S. M., Ragan, E. P., \& Whitton, S. W. (2010). The premarital communication roots of marital distress and divorce: The first five years of marriage. Journal of Family Psychology, 24(3), 289-98. doi: https://doi. org/10.1037/a0019481.

Miller, S., Nunnally, E. W., \& Wackman, D. B. (1976). A communication training program for couples. Social Casework, 57(1), 9-18. https://doi.org/10.1177/104438947605700102

Mosmann, C., Wagner, A., \& Féres-Carneiro, T. (2006). Qualidade conjugal: mapeando conceitos, Paidéia, 16(35), 315-325. https://doi.org/10.1590/S0103-863X2006000300003.

Murta, S. G., Rodrigues, A. C., Rosa, I. de O., \& Paulo, S. G. de. (2012). Avaliação de um programa psicoeducativo de transição para a parentalidade. Paidéia, 22(53), 403-412. https://doi. org/10.1590/S0103-863X2012000300012

Neff, L. A., \& Broady, E. F. (2011). Stress resilience in early marriage: Can practice make perfect? Journal of Personality and Social Psychology, 101(5), 1050-1067. https://doi. org/10.1037/a0023809

Neumann, A. P., Wagner, A., \& Remor, E. (2018). Couple relationship education program "Living as Partners": Evaluation of effects on marital quality and conflict. Psicologia: Reflexão $e$ Crítica, 31, 26. https://dx.doi.org/10.1186/s41155-018-0106-z

Norgren, M. de B. P., Souza, R. M. de, Kaslow, F., Hammerschmidt, H., \& Sharlin, S. A. (2004). Satisfação conjugal em casamentos de longa duração: Uma construção possível. Estudos de Psicologia (Natal), 9(3), 575-584. https://doi.org/10.1590/ S1413-294X2004000300020

Ooms, T. (2005). The new kid on the block: What is marriage education and does it work?. Washington: Center for Law and Social Policy (CLASP). Retrieved from https://vawnet. org/material/new-kid-block-what-marriage-education-anddoes-it-work

Owen, J., Manthos, M., \& Quirk, K. (2013). Dismantling study of prevention and relationship education program: The effects of a structured communication intervention. Journal of Family Psychology, 27(2), 336-341. https://doi.org/10.1037/a0031597

Petch, J. F., Halford, W. K., Creedy, D. K., \& Gamble, J. (2012a). A randomized controlled trial of a couple relationship and coparenting program (Couple CARE for Parents) for highand low-risk new parents. Journal of Consulting and Clinical Psychology, 80(4), 662-73. https://doi.org/10.1037/a0028781

Petch, J., Halford, W. K., Creedy, D. K., \& Gamble, J. (2012b). Couple relationship education at the transition to parenthood: A window of opportunity to reach high-risk couples. Family Process, 51(4), 498-511. https://doi.org/10.1111/j.15455300.2012.01420.x
Pihet, S., Bodenmann, G., Cina, A., Widmer, K., \& Shantinath, S. D. (2007). Can prevention of marital distress improve well-being? Results of a 1-year longitudinal study. Clinical Psychology and Psychotherapy, 14, 79-88. https://doi.org/10.1002/cpp.522

Quirk, K., Strokoff, J., Owen, J. J., France, T., \& Bergen, C. (2014). Relationship education in community settings: Effectiveness with distressed and non-distressed low-income racial minority couples. Journal of Marital and Family Therapy, 40(4), 442-53. https://doi.org/10.1111/jmft.12080

Rauer, A. J., Adler-Baeder, F., Lucier-Greer, M., Skuban, E., Ketring, S. A., \& Smith, T. (2014). Exploring processes of change in couple relationship education: Predictors of change in relationship quality. Journal of Family Psychology, 28(1), 65-76. https://doi.org/10.1037/a0035502

Rhoades, G. K. (2015). The effectiveness of the Within Our Reach Relationship Education Program for Couples: Findings from a federal randomized trial. Family Process, 54(4), 672-685. https://doi.org/10.1111/famp.12148

Owen, J. and Rhoades, G. K. (2012), Reducing interparental conflict among parents in contentious child custody disputes: An initial investigation of the Working Together Program. Journal of Marital and Family Therapy, 38, 542-555. https:// doi.org/10.1111/j.1752-0606.2010.00215.x

Rhoades, G. K., \& Stanley, S. M. (2011). Using individual-oriented relationship education to prevent family violence. Journal of Couple \& Relationship Therapy, 10(2), 185-200. https://doi. org/10.1080/15332691.2011.562844

Rhoades, G. K., Stanley, S. M., \& Markman, H. J. (2009). Working with cohabitation in relationship education and therapy. Journal of Couple \& Relationship Therapy, 8(2), 95-112. https://doi. org/10.1080/15332690902813794

Ripley, J. S., \& Worthington, E. L., Jr. (2002). Hope-focused and forgiveness-based group interventions to promote marital enrichment. Journal of Counseling \& Development, 80(4), 452-463. http://doi.org/10.1002/j.1556-6678.2002.tb00212.x

Sardinha, A., Falcone, E. M. O., \& Ferreira, M. C. (2009). As relações entre a satisfação conjugal e as habilidades sociais percebidas no cônjuge. Psicologia: Teoria e Pesquisa, 25(3), 395-402. http://doi.org/10.1590/S0102-37722009000300013.

Sbarra, D. A. (2015). Divorce and health: Current trends and future directions. Psychosomatic Medicine, 77(3), 227-236. https:// doi.org/10.1097/PSY.0000000000000168

Schulz, M. S., Cowan, C. P., \& Cowan, P. A. (2006). Promoting healthy beginnings: A randomized controlled trial of a preventive intervention to preserve marital quality during the transition to parenthood. Journal of Consulting and Clinical Psychology, 74(1), 20-31. https://doi.org/10.1037/0022006X.74.1.20

Scorsolini-Comin, F. \& Santos, M. A. (2009). Casar e ser feliz: Mapeando a mensuração da satisfação conjugal. Psico, 40(4), 430-437

Scott, S. B., Rhoades, G. K., Stanley, S. M., Allen, E. S., \& Markman, H. J. (2013). Reasons for divorce and recollections of premarital intervention: Implications for improving relationship education. Couple \& Family Psychology, 2(2), 131-145. https://doi.org/10.1037/a0032025

Spanier, G. B. (1976). Measuring dyadic adjustment: New scales for assessing the quality of marriage and similar dyads. Journal of Marriage and the Family, 38(1), 15-28.

Stanley, S. M., Allen, E. S., Markman, H. J., Rhoades, G. K., \& Prentice, D. L. (2010). Decreasing divorce in army couples: Results from a randomized controlled trial using PREP for strong bonds. Journal of Couple Relationship Therapy, 9(2), 149-160. https://doi.org/10.1080/15332691003694901

Stanley, S. M., Amato, P. R., Johnson, C. A., \& Markman, H. J. (2006). Premarital education, marital quality, and marital stability: Findings from a large, random household survey. 
Journal of Family Psychology, 20(1), 117-126. https://doi. org/10.1037/0893-3200.20.1.117

Stanley, S. M., Rhoades, G. K., Loew, B. A., Allen, E. S., Carter, S., Osborne, L. J., ... Markman, H. J. (2014). A randomized controlled trial of relationship education in the U.S. Army: 2-year outcomes. Family Relations, 63(4), 484-495. https:// doi.org/10.1111/fare. 12083

Thuen, F., \& Laerum, K. T. (2005). A public/private partnership in offering relationship education to the Norwegian population. Family Process, 44(2), 175-185. Retrieved from http://www. ncbi.nlm.nih.gov/pubmed/16013744

Vedes, A. M., Lind, W., \& Lourenço, M. (2011). Fundamentos para o desenho de estratégias de prevenção para a promoção da satisfação e da resiliência conjugal. Psicologia, 25(1), 91-112.

Visvanathan, P. D., Richmond, M., Winder, C., \& Koenck, C. H. (2015). Individual-oriented relationship education: An evaluation study in community-based settings. Family Process, 54(4), 686-702. https://doi.org/10.1111/famp.12116

Wadsworth, M. E., \& Markman, H. J. (2013). Where's the action? Understanding what works and why in relationship education. Behavior Therapy, 43(1), 99-112. https://doi.org/10.1016/j. beth.2011.01.006

Wagner, A., Mosmann, C., \& Falcke, D. (2015). Viver a dois: Oportunidades e desafios da conjugalidade. São Leopoldo: Sinodal.

Walsh, F. (2005). Fortalecendo a resiliência familiar. São Paulo: Roca.
Wang, P. (1991). Pre-marital education bears fruits. China Population Today, 8(4), 17. Retrieved from http://www.ncbi. nlm.nih.gov/pubmed/12317272

Whitton, S. W., Weitbrecht, E. M., Kuryluk, A. D., \& Hutsell, D. W. (2016). A randomized waitlist-controlled trial of culturally sensitive relationship education for male same-sex couples. Journal of Family Psychology, 30(6), 763-768. https://doi. org/10.1037/fam0000199

Williamson, H. C., Altman, N., Hsueh, J., \& Bradbury, T. N. (2016). Effects of relationship education on couple communication and satisfaction: A randomized controlled trial with low-income couples. Journal of Consulting and Clinical Psychology, 84(2), 156-166. https://doi.org/10.1037/ccp0000056

Williamson, H. C., Hammett, J. F., Ross, J. M., Karney, B. R., \& Bradbury, T. N. (2018). Premarital education and later relationship help-seeking. Journal of Family Psychology, 32(2), 276-281. https://doi.org/10.1037/fam0000383.

Wood, R. G., McConnell, S., Moore, Q., Clarkwest, A., \& Hsueh, J. A. (2010). Strengthening unmarried parents' relationships: The early impacts of building strong families. Princeton, NJ: Mathematica Policy Research.

Yao, Z. (1990). A serial surveillance for premarital health care. China Population Research Leads, 9, 1. Retrieved from http:// www.ncbi.nlm.nih.gov/pubmed/12342915

Yoo, H., Bartle-Haring, S., Day, R. D., \& Gangamma, R. (2014). Couple communication, emotional and sexual intimacy, and relationship satisfaction. Journal of Sex and Marital Therapy, 40(4), 275-293. https://doi.org/10.1080/009262 3X.2012.751072. 